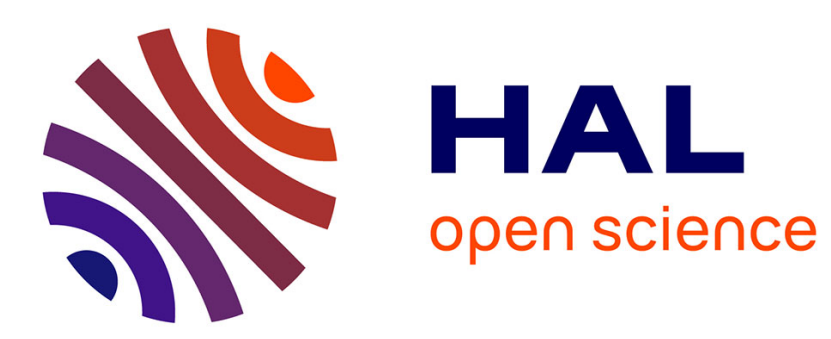

\title{
Numerical simulations of a rising drop with shape oscillations in the presence of surfactants
}

Antoine Rémy Piedfert, Benjamin Lalanne, Olivier Masbernat, Frédéric Risso

\section{To cite this version:}

Antoine Rémy Piedfert, Benjamin Lalanne, Olivier Masbernat, Frédéric Risso. Numerical simulations of a rising drop with shape oscillations in the presence of surfactants. Physical Review Fluids, 2018, 3 (10), pp.103605. 10.1103/PhysRevFluids.3.103605 . hal-02057842

\section{HAL Id: hal-02057842 \\ https://hal.science/hal-02057842}

Submitted on 5 Mar 2019

HAL is a multi-disciplinary open access archive for the deposit and dissemination of scientific research documents, whether they are published or not. The documents may come from teaching and research institutions in France or abroad, or from public or private research centers.
L'archive ouverte pluridisciplinaire HAL, est destinée au dépôt et à la diffusion de documents scientifiques de niveau recherche, publiés ou non, émanant des établissements d'enseignement et de recherche français ou étrangers, des laboratoires publics ou privés. 


\section{OATAO \\ Open Archive Toulouse Archive Ouverte}

\section{Open Archive Toulouse Archive Ouverte (OATAO)}

OATAO is an open access repository that collects the work of Toulouse researchers and makes it freely available over the web where possible.

This is an author-deposited version published in: http://oatao.univ-toulouse.fr/ Eprints ID: 22905

To link to this article : DOI:10.1103/PhysRevFluids.3.103605

URL : https://doi.org/10.1103/PhysRevFluids.3.103605

To cite this version: Piedfert, Antoine Rémy and Lalanne, Benjamin and Masbernat, Olivier $\stackrel{5}{\omega}$ and Risso, Frédéric Numerical simulations of a rising drop with shape oscillations in the presence of surfactants. (2018) Physical Review Fluids, 3 (10). 1-22. ISSN 2469-990X

Any correspondence concerning this service should be sent to the repository administrator:

staff-oatao@listes-diff.inp-toulouse.fr 


\title{
Numerical simulations of a rising drop with shape oscillations in the presence of surfactants
}

\author{
Antoine Piedfert, ${ }^{1,2,3}$ Benjamin Lalanne, ${ }^{1,3, *}$ Olivier Masbernat, ${ }^{1,3}$ and Frédéric Risso ${ }^{2,3}$ \\ ${ }^{1}$ Laboratoire de Génie Chimique, Université de Toulouse, CNRS, INPT, UPS, Toulouse, France \\ ${ }^{2}$ Institut de Mécanique des Fluides de Toulouse (IMFT), Université de Toulouse, CNRS, Toulouse, France \\ ${ }^{3}$ FERMAT, Université de Toulouse, CNRS, INPT, INSA, UPS, Toulouse, France
}

(Received 31 May 2018; published 25 October 2018)

\begin{abstract}
The effect of insoluble surfactants on the dynamics of a drop immersed in another liquid has been numerically investigated. The interface is captured by means of the Level-Set method, and the evolution of the surfactant concentration $\Gamma$ is solved along the deformable interface. The Marangoni stress resulting from tangential gradients of $\Gamma$ has been implemented in the Ghost Fluid method, allowing one to compute the viscous-stress jump at the interface. The numerical model is first evaluated in the case of an oscillating droplet at mode 2 . Numerical results accurately reproduce the shape-oscillation dynamics and demonstrate that the presence of surfactants has a strong impact on the damping rate. This effect, however, does not evolve monotonically with $\Gamma$ but rather follows the evolution of the maximal value of the gradient of $\Gamma$ that develops over the drop surface. The case of rising spherical droplets is next considered, where the distribution of surfactant at steady state results from the balance between tangential advection towards the rear of the droplet and the upward Marangoni flux. It is shown that the droplet velocity matches that of a solid particle, although a significant part of the interface is still surfactant free (around $30 \%$ ). The gradient of $\Gamma$ induced by the rising motion does not evolve monotonically with $\Gamma$ as a result of the competition between Marangoni stress and the shear stress in the external boundary layer. Finally, the study of the case combining shape oscillations and rising motion shows that the rising motion does not influence the oscillation dynamics in the limit of low Weber number. The particular case of a droplet oscillating as a clean droplet (no change of eigenmodes) and rising as a fully contaminated droplet could be exhibited, in agreement with previous experimental observations.
\end{abstract}

DOI: 10.1103/PhysRevFluids.3.103605

\section{INTRODUCTION}

In multiphase flows involving droplets or bubbles immersed in a liquid, such as in emulsification processes or bubble columns, contaminants or surfactants are often present at the interfaces, either in controlled bulk concentrations in order, for example, to ensure the emulsion stability or as inadvertent traces. However, it is well known that only a residual amount of surfactant is sufficient to drastically change the droplet or bubble dynamics [1] compared to a case with clean interfaces. Indeed, the rising or settling velocity of a droplet was measured to be smaller [2,3] than the prediction of the Hadamard-Rybczynski theory for clean interfaces; this was explained for the first time by Frumkin and Levich [4] as a consequence of the flow caused at the interface by the existence of surface tension gradients, which is known as the Marangoni effect. Other consequences of the presence of surfactants can be observed in the drop or bubble dynamics, such as changes in the

*Corresponding author: Benjamin.Lalanne@ensiacet.fr 
rising path regime [5], a noticeable decrease of heat and mass transfer rates between the continuous and the dispersed phase [6,7], and a prevention of drop coalescence [8].

Generally, it is very difficult to access interfacial quantities such as the surface concentration of adsorbed surfactants or the tangential velocity at the interface, even if, recently, Hokosawa et al. [9] could evaluate them by means of spatio-temporal filter velocimetry for rising droplets in the Stokes regime. Then direct numerical simulations can be employed as a complementary tool to analyze experimental results and to investigate the effect of surfactants on the droplet or bubble dynamics, in particular at a large Reynolds number. A few direct simulation works exist in the presence of surfactants, such as those of Cuenot et al. [10] with a boundary-fitted method, Drumright-Clarke and Renardy [11] and James and Lowengrub [12] with the Volume of Fluid method, Xu and Zhao [13] and Cleret de Langavant et al. [14] with the Level-Set method, or Muradoglu and Tryggvason [15] with the Front-Tracking method. In this paper, the Level-Set method described in Lalanne et al. [16] is extended to account for the presence of insoluble surfactants at gas-liquid or liquid-liquid interfaces.

The objective is to use numerical simulations in order to investigate the effect of surfactants on the shape-oscillation dynamics of a droplet. This problem is of particular interest since it corresponds to experiments of interfacial rheology measurement. Such experiments consist in analyzing the droplet dynamics in response to known perturbations, either in the case of forced volume oscillations for a droplet attached to a capillary tip as in Abi Chebel et al. [17] or in the case of a freely rising droplet for which the shape oscillates, as in Lalanne et al. [18]; these experiments are performed to determine the characteristic interface timescales. This paper focuses on the latter configuration. Hence, the objective is to quantify the effect of contamination by surfactants on the coupling between the oscillating motion and the rising motion of a droplet.

Previous theoretical and experimental works exist for the problem of linear shape oscillations of a droplet or bubble, but only a few studies have been dedicated to tackling either the effect of a contaminated interface or that of a rising motion on the shape-oscillation dynamics. In this problem, the fluid system is characterized by density $\left(\widehat{\rho}=\frac{\rho_{i}}{\rho_{o}}\right)$ and viscosity $\left(\widehat{\mu}=\frac{\mu_{i}}{\mu_{o}}\right)$ ratios of the two phases, $\rho_{o}$ and $\rho_{i}$ being respectively the density of outer and inner phase and $\mu_{o}$ and $\mu_{i}$ their respective dynamic viscosities. The shape-oscillating motion is characterized by a Reynolds number of oscillation $\mathrm{Re}_{\mathrm{osc}}=\frac{\rho_{o} \sqrt{\frac{\sigma_{e}}{\rho_{o} R^{3}}} R^{2}}{\mu_{o}}$, with $R$ the radius of the sphere of the same volume as that of the droplet and $\sigma_{e}$ the interfacial tension between the two phases at equilibrium. In the presence of gravity, a sliding motion exists between the droplet and the continuous phase, characterized at steady state by a rising Reynolds number $\operatorname{Re}_{\text {asc }}=\frac{\rho_{o} V_{\infty} d}{\mu_{o}}, d(=2 R)$ and $V_{\infty}$ being respectively the diameter and the terminal velocity of the undeformed droplet. In the presence of surfactants, the number of parameters involved in the problem drastically increases, but in the dilute limit and neglecting transfers of surfactant between the interface and the bulk phases, the parameter required to describe their effects is the Gibbs elasticity; its nondimensional form is the elasticity number $E$, which compares the intensity of variation of interfacial tension in response to a variation of surface area $S$ over the interfacial tension $\sigma_{e}$ at equilibrium: $E=\frac{S \frac{d \sigma}{d S}}{\sigma_{e}}$.

In the absence of rising motion and without surfactant $\left(\mathrm{Re}_{\mathrm{asc}}=0, E=0\right)$, the problem has been studied theoretically by Rayleigh [19], Lamb [20], Miller and Scriven [21], and Prosperetti [22] in the case of oscillations of low amplitude. The mathematical solution of the linearized problem shows that the interface dynamics can be described by a series of modes, each of them being associated to a given shape evolving in time with an eigenfrequency $\omega_{n}$ and a damping rate $\beta_{n}$.

In the presence of gravity and without surfactant, Lalanne et al. [23] have shown through numerical simulations that the rising motion can change the values of $\omega_{n}$ and $\beta_{n}$ provided the Weber number based on the rising velocity $\mathrm{We}=1 / 2\left(\mathrm{Re}_{\mathrm{asc}} / \mathrm{Re}_{\mathrm{osc}}\right)^{2}$ becomes larger than unity: Eigenmodes of oscillation are indeed modified when the average droplet (or bubble) shape is significantly deformed due to the rise velocity, with frequencies that decrease slightly compared to the nonrising case; for drops, the damping rate increases significantly with We, whereas for bubbles, 
at the terminal stage, the damping rate is close to the value without rising motion. However, for millimeter-size droplets or submillimeter-size bubbles in common fluids, the Weber number remains generally small; then the rising droplet or bubble oscillates around a spherical shape, and $\omega_{n}$ and $\beta_{n}$ are very close to the theoretical predictions in the absence of gravity, as illustrated in Lalanne et al. [18] with experimental data.

In the presence of surfactants at a dilute concentration and without gravity $\left(\operatorname{Re}_{\text {asc }}=0\right)$, Miller and Scriven [21] and Lu and Apfel [24] have addressed theoretically the linearized problem, providing general expressions that can be computed to derive $\omega_{n}$ and $\beta_{n}$. A few experimental results also exist (Tian et al. [25], Corti et al. [26], Asaki et al. [27]), which show that the damping rate of the shape oscillations can be strongly increased compared to the perfectly clean case and that its evolution with the surfactant concentration is nonmonotonic, some authors reporting the observation of a maximum of damping rate at a given concentration.

Finally, in the presence of both surfactants and gravity, Abi Chebel et al. [28] have measured the frequency and the damping rate of several modes in contaminated cases, observing that the effect of contamination becomes negligible for higher order modes. As far as we know, there is no numerical simulations of this complete problem, although the numerical approach is well suited to study separately the independent and coupled influences of the presence of both surfactant and gravity effects, as addressed in the present paper.

In this study, three main assumptions are taken: (i) surfactants are insoluble, i.e., there is no mass exchange of surfactants between the interface and bulk phases, (ii) the surface concentration of surfactants remains very low compared to the concentration of saturation at the interface, and (iii) in the cases where the rising motion of the droplet is considered, the Weber number is maintained very low, ensuring that the drop average shape remains spherical, as is the case with millimeter-size droplets in common liquids. Assumption (ii) is consistent with both considering a linear equation of state to relate the local interfacial tension $\sigma$ to the local surface concentration $\Gamma$, and the Marangoni stress as the only force due to the presence of surfactants at the interface; intrinsic elasticity or surface viscosity forces resulting from interactions between adsorbed molecules are not addressed in this work.

Section II presents the equations that are solved for this problem, and Sec. III introduces the numerical methods implemented in our in-house code DIVA. Section IV presents the validation of the proposed model on simple test cases. Section V successively addresses the cases of a shape-oscillating contaminated droplet in the absence of gravity $\left(\operatorname{Re}_{\mathrm{asc}}=0\right)$, of a freely rising contaminated droplet without shape oscillations $(E=0)$, and of the coupling between shapeoscillating and rising motions. Our main conclusions are summarized in Sec. VI.

\section{PHYSICAL FORMULATION FOR INSOLUBLE SURFACTANTS}

The physical formulation of the problem is based on the one-fluid model for two incompressible fluids. The velocity of either fluid at any given point $\vec{x}$ and time $t$ is noted $\vec{v}(\vec{x}, t)$, and pressure $P(\vec{x}, t)$. The Navier-Stokes equations are expressed as follows:

$$
\begin{gathered}
\rho\left[\frac{\partial \vec{v}}{\partial t}+(\vec{v} \cdot \vec{\nabla}) \vec{v}\right]=-\vec{\nabla} P+\vec{\nabla} \cdot(\mu \vec{\nabla} \vec{v})+\rho \vec{g}, \\
\vec{\nabla} \cdot \vec{v}=0,
\end{gathered}
$$

where $\rho$ and $\mu$ are the density and the dynamic viscosity of the considered fluid respectively, and $\vec{g}$ is the gravity acceleration.

When the interface is surfactant-free, the interfacial tension is uniform and noted $\sigma_{c}$. In the presence of adsorbed molecules, the interfacial tension $\sigma(\vec{x}, t)$ between the two fluids varies in both space and time and depends on the surface concentration $\Gamma(\vec{x}, t)$ of surfactants. In the dilute case, the Henry equation of state gives the relation between $\sigma$ and $\Gamma$ :

$$
\sigma=\sigma_{c}-\bar{R} T \Gamma
$$


$T$ being the temperature and $\bar{R}$ the ideal gas constant. In this work, insoluble surfactants are considered, thus their presence is accounted for only at the interface: this assumption is equivalent to considering that the characteristic times of the adsorption and desorption processes between the bulk phases and the interface are very large compared to the timescales of both the shape oscillations and the rising motion. Then the number of molecules of surfactants at the interface remains constant: $\int_{S(t)} \Gamma d S=M_{0}$, where $S(t)$ is the total surface of the interface and $M_{0}$ the mole number. At each instant, the average surface concentration of the droplet is noted $\bar{\Gamma}(t)$. Note that, due to mole conservation $[d(\bar{\Gamma} S)=0]$ and dilute regime assumption [Eq. (3)], the elasticity number can be written as $E=\frac{\bar{R} T \bar{\Gamma}_{e}}{\sigma_{e}}$.

Due to the fluid flow at the interface, surfactants are advected along the (deformable) drop surface, and mass conservation reads [29,30]:

$$
\frac{\partial \Gamma}{\partial t}+\vec{\nabla}_{s} \cdot(\Gamma \vec{v})=D_{s} \vec{\nabla}_{s}^{2} \Gamma
$$

where $\vec{\nabla}_{s}=(\overrightarrow{\vec{I}}-\vec{n} \otimes \vec{n}) \vec{\nabla}$ is the surface gradient operator (with $\vec{n}$ the normal vector to the interface) and $D_{s}$ the surface diffusion coefficient of surfactants. In the limit of high surface Péclet number considered in this study, the right-hand side diffusion term of Eq. (4) can be safely neglected. The transport equation of $\Gamma$ can be rewritten following

$$
\frac{\partial \Gamma}{\partial t}+\vec{v}_{t} \cdot \vec{\nabla}_{s} \Gamma+\Gamma \vec{\nabla}_{s} \cdot \vec{v}=0
$$

where $\vec{v}_{t}$ is the local tangential velocity at the interface. The third term of Eq. (5) can be itself decomposed into two contributions, $\vec{\nabla}_{s} \cdot \vec{v}=\vec{\nabla}_{s} \cdot \vec{v}_{t}+\left(\vec{\nabla}_{s} \cdot \vec{n}\right) v_{n}$, by denoting $v_{n}$ the normal component of the velocity at the interface. Equation (5) therefore indicates that variations of $\Gamma$ are due to three contributions: (i) the advective transport by the tangential velocity $\vec{v}_{t}$ along the interface, (ii) the nonzero divergence of the surface flow, and (iii) the deformation of the interface due to the normal velocity component $v_{n}$.

The coupling between internal and external bulk flows is modeled through boundary conditions at the interface. Tangential and normal components of velocity are continuous across the interface. However, normal stresses are discontinuous, with a jump due to the Laplace pressure ([?] used for interfacial jump notation):

$$
[\vec{n} \cdot \overrightarrow{\vec{\tau}} \cdot \vec{n}]=\sigma \kappa,
$$

where $\overrightarrow{\vec{\tau}}=-P \overrightarrow{\vec{I}}+2 \mu \overrightarrow{\vec{D}}$ is the stress tensor, $\overrightarrow{\vec{D}}=\frac{1}{2}\left(\vec{\nabla} \vec{v}+\vec{\nabla} \vec{v}^{t}\right)$ the rate of deformation tensor, and $\kappa$ the local mean curvature of the interface $(\overrightarrow{\vec{I}}$ is the identity tensor).

Surfactant concentration gradients $\left(\vec{\nabla}_{s} \Gamma\right)$ along the interface generate a tangential stress jump through gradients of surface tension:

$$
[\vec{t} \cdot \overrightarrow{\vec{\tau}} \cdot \vec{n}]=-\vec{t} \cdot \vec{\nabla}_{s} \sigma
$$

where $\vec{t}$ is a tangential vector to the interface. This Marangoni stress tends to smoothen the gradients of $\Gamma$.

\section{NUMERICAL FORMULATION}

The method implemented in the in-house DIVA code for two-phase flows is explained in detail in Lalanne et al. [16]. It runs direct numerical simulations in an axisymmetric domain by solving the Navier-Stokes equations in the framework of one-fluid formulation. The interface is captured in a fixed Eulerian mesh using the Level-Set method. In the framework of sharp interface methods, jump conditions at the interface are accounted for by means of a Ghost Fluid method, following the work of Kang et al. [31], in order to deal with viscosity, pressure, and tangential stress discontinuities. 
All equations are implemented using the finite volume method, where temporal derivatives are discretized using a Runge-Kutta scheme of order 2, convective terms by a WENO scheme of order 5 , and all the other spatial derivatives using a central scheme of order 2.

We briefly present next the outlines of these methods, with a highlight on developments related to the implementation of surfactant interfacial transport.

\section{A. The Level-Set method}

The Level-Set method [32] represents a deformable and moving interface in a fixed grid with the help of a scalar function $\phi$ which is equal to the (normal) signed distance between the considered point of the domain and the interface: by definition, $\phi=0$ at the interface, and $\phi$ is defined to be negative inside the drop and positive outside. The Level-Set function $\phi$ is advected by the flow like a passive scalar:

$$
\frac{\partial \phi}{\partial t}+\vec{v} \cdot \vec{\nabla} \phi=0
$$

Between two iterations, an algorithm of redistanciation is used to ensure that $\phi$ remains a distance function, which consists in solving the following equation at each time step over a fictive time $\tau$ :

$$
\frac{\partial \widetilde{\phi}}{\partial \tau}=\operatorname{sign}(\phi)(1-|\vec{\nabla} \widetilde{\phi}|),
$$

where $\widetilde{\phi}$ is the reinitialized Level-Set function such as $\widetilde{\phi}(\vec{x}, t, \tau=0)=\phi(\vec{x}, t)$, and the sign function is smoothed numerically ( $\Delta x$ is the size of the lowest mesh cell):

$$
\operatorname{sign}(\phi)= \begin{cases}-1 & \text { if } \phi<-\Delta x \\ \frac{\phi}{\sqrt{\phi^{2}+\Delta x^{2}}} & \text { if }|\phi| \leqslant \Delta x \\ 1 & \text { if } \phi>\Delta x\end{cases}
$$

Normal vector and curvature are then calculated as follows: $\vec{n}=\vec{\nabla} \widetilde{\phi} /\|\vec{\nabla} \widetilde{\phi}\|$ and $\kappa=\vec{\nabla} \cdot \vec{n}$.

\section{B. The Ghost Fluid method}

In order to estimate properly the jump conditions at the interface, a Ghost Fluid method, originally proposed by Kang et al. [31] and presented in Lalanne et al. [16], labeled the Ghost Fluid primitive viscous method, has been implemented. It consists in creating ghost values of the discontinuous variables across the interface in order to make them accurately differentiable in the neighborhood of the interface. Extension of these variables is achieved using the analytical expression of the jump conditions. We have used this method in order to impose pressure and viscous stress jumps across the interface. According to Kang et al. [31], jump due to viscosity is expressed into the form of a viscous flux jump matrix in the absence of surfactant effects; in this work, we have included the Marangoni stress in the viscous stress jump expression, leading to the following two-dimensional jump matrix at the interface:

$$
\begin{aligned}
{[\mu \vec{\nabla} \vec{v}]=} & {[\mu]\left(\begin{array}{c}
\vec{\nabla} v_{x} \\
\vec{\nabla} v_{y}
\end{array}\right)\left(\begin{array}{c}
\overrightarrow{0} \\
\vec{t}
\end{array}\right)^{T}\left(\begin{array}{c}
\overrightarrow{0} \\
\vec{t}
\end{array}\right)+[\mu] \vec{n} \vec{n}\left(\begin{array}{c}
\vec{\nabla} v_{x} \\
\vec{\nabla} v_{y}
\end{array}\right) \vec{n} \vec{n}-[\mu]\left(\begin{array}{c}
\overrightarrow{0} \\
\vec{t}
\end{array}\right)^{T}\left(\begin{array}{c}
\overrightarrow{0} \\
\vec{t}
\end{array}\right)\left(\begin{array}{l}
\vec{\nabla} v_{x} \\
\vec{\nabla} v_{y}
\end{array}\right)^{T} \vec{n} \vec{n} } \\
& -\left(\vec{\nabla}_{s} \sigma \cdot \vec{t}\right) \vec{t} \otimes \vec{n} .
\end{aligned}
$$

The last term of Eq. (10) is the Marangoni stress term, acting in the tangential direction. The Ghost Fluid method for the Marangoni term has also been used in the work of Lervag et al. [33] and Hayashi and Tomiyama [34]. 


\section{Extension method of surface concentration}

In order to compute surface gradients of $\sigma$ along the interface, which does not contain any mesh points, a numerical extension $\Gamma$ of the surface concentration $\Gamma$ is introduced and defined in the whole domain, as initially proposed by Xu and Zhao [13]. $\widetilde{\Gamma}$ is computed from $\Gamma$ as a zero gradient field in the normal direction to the interface, by solving the following equation:

$$
\frac{\partial \widetilde{\Gamma}}{\partial \widehat{t}}+\operatorname{sign}(\phi) \vec{n} \cdot \vec{\nabla} \widetilde{\Gamma}=0
$$

at each time step, over a fictitious time $\widehat{t}$; in this way, after a few iterations, $\widetilde{\Gamma}$ satisfies $\vec{n} \cdot \vec{\nabla} \widetilde{\Gamma}=0$ in the neighborhood of the interface (over a distance equal to four times the local mesh element).

Then the transport equation of the surface concentration [Eq. (5)] is easily solved in the whole domain, due to the property $\nabla_{s} \widetilde{\Gamma}=\nabla \widetilde{\Gamma}$. Note that Eq. (5) is computed using the identity $\vec{\nabla}_{s} \cdot \vec{v}=$ $-\vec{n} \cdot \vec{\nabla} \vec{v} \cdot \vec{n}$, valid for an incompressible flow.

\section{VALIDATION}

In order to validate the implementation of the effects of surfactants, several test cases have been simulated. These test cases have been chosen to evaluate the numerical resolution of advection of surfactants on a deformable interface given by Eq. (5), the method of extension of the surface concentration given by Eq. (11), and the Marangoni term in the jump of viscous stresses in Eq. (10).

\section{A. Accuracy of the equation of transport}

The first test case considers the expansion in the radial direction of a two-dimensional rotating cylinder (of infinite length). The flow imposed in the domain of calculation is the sum of a purely rotating flow and of the flow generated by a point source located at the center of the cylinder. In polar coordinates $(r, \theta)$, the fluid velocity field is given by

$$
\vec{v}=\omega r \vec{e}_{\theta}+\frac{Q}{r} \vec{e}_{r},
$$

in which the flow rate per unit length $Q$ and the angular velocity $\omega$ are constant. The surface concentration profile of surfactants is initialized at the interface as

$$
\Gamma(\theta, t=0)=\Gamma_{m}(0)\left[\frac{1+\cos (2 \theta)}{2}\right] .
$$

The profile of $\Gamma$ evolves because of both the tangential velocity and the radial expansion, as described by Eq. (5). At each instant $t$, the maximum value of $\Gamma_{m}(t)$ over $\theta$ can be determined from the conservation of mole number, $2 \pi R(0) \Gamma_{m}(0)=2 \pi R(t) \Gamma_{m}(t)$, where $R(t)$ is the radius of the cylinder at time $t$. Knowing the imposed normal velocity of the growing interface $d R / d t=Q / R$, the profile of surface concentration is given by

$$
\Gamma(\theta, t)=\frac{\Gamma_{m}(0)}{\sqrt{1+\frac{2 Q}{R(0)^{2}} t}}\left\{\frac{1+\cos [2(\theta-\omega t)]}{2}\right\} .
$$

According to the imposed flow [Eq. (12)], the numerical code calculates the time evolution of the profile of $\Gamma$, which can be compared to the exact theoretical expression [Eq. (13)]. The simulation of this problem is performed using several meshes of $6,12,24$, and 48 nodes per initial cylinder radius, during the time $t_{f}$ required to accomplish four revolutions. In this time interval, the flow rate makes double the initial radius, leading to a theoretical final value of $\Gamma_{m}\left(t_{f}\right)=1 / 2 \Gamma_{m}(0)$. In Fig. 1 the profiles of $\Gamma$ obtained with the different meshes are observed to converge accurately towards the analytical solution, with a full convergence reached for a mesh refinement lying between 24 and 48 nodes per initial radius. It can be observed that the advection of $\Gamma$ by the rotating motion 




FIG. 1. Test case of interface rotation and expansion: profiles of $\Gamma$ for different meshes at $t=t_{f}$ with 6,12 , 24, and 48 nodes per radius, compared to the analytical result of Eq. (13) with $Q=3.8197 \times 10^{-10} \mathrm{~m}^{2} \mathrm{~s}^{-1}$, $R(0)=4 \times 10^{-5} \mathrm{~m}, \omega=4 \mathrm{rad} / \mathrm{s}, t_{f}=2 \pi \mathrm{s}$.

is accurately solved even with a resolution of 12 nodes per initial radius, whereas the change of $\Gamma$ due to surface variation requires a minimum of 24 nodes per initial radius to be accurate enough [validated by the value of $\left.\Gamma_{m}\left(t_{f}\right)\right]$.

\section{B. Marangoni stress jump}

The second test case aims at validating the numerical implementation of the Marangoni stress jump using the Ghost Fluid method. We consider the case of a drop placed in a uniform temperature gradient in the absence of gravity, leading to an interfacial tension gradient and hence to the buildup of interface tangential stresses. Young et al. [35] have calculated theoretically the rising velocity at steady state that results from these Marangoni stresses, by linearizing the problem and solving the Stokes equations around a spherical droplet. In the axisymmetric simulation, a gradient of surface tension is imposed in the $z$ direction following

$$
\sigma(z)=\sigma_{e}\left(1-\frac{E z}{l_{z}}\right)
$$

where $l_{z}$ represents the size of the domain in the vertical direction and $E$ is the elasticity number. In order to simulate the rising motion, only the Navier-Stokes equations and the boundary conditions given by Eqs. (6) and (7) are solved until steady state is reached, in a regime where both the Reynolds and Weber numbers remain very low, their respective values being given in the caption of Fig. 2 . The time evolution of the rising velocity of the drop is displayed in Fig. 2(a) for several meshes of 16, 32, and 64 nodes per radius, the velocity being normalized by the theoretical prediction of Young et al. [35], which is

$$
V_{\infty}=\frac{2 E \sigma_{e}}{l_{z}\left(6 \mu_{o}+9 \mu_{i}\right)}
$$



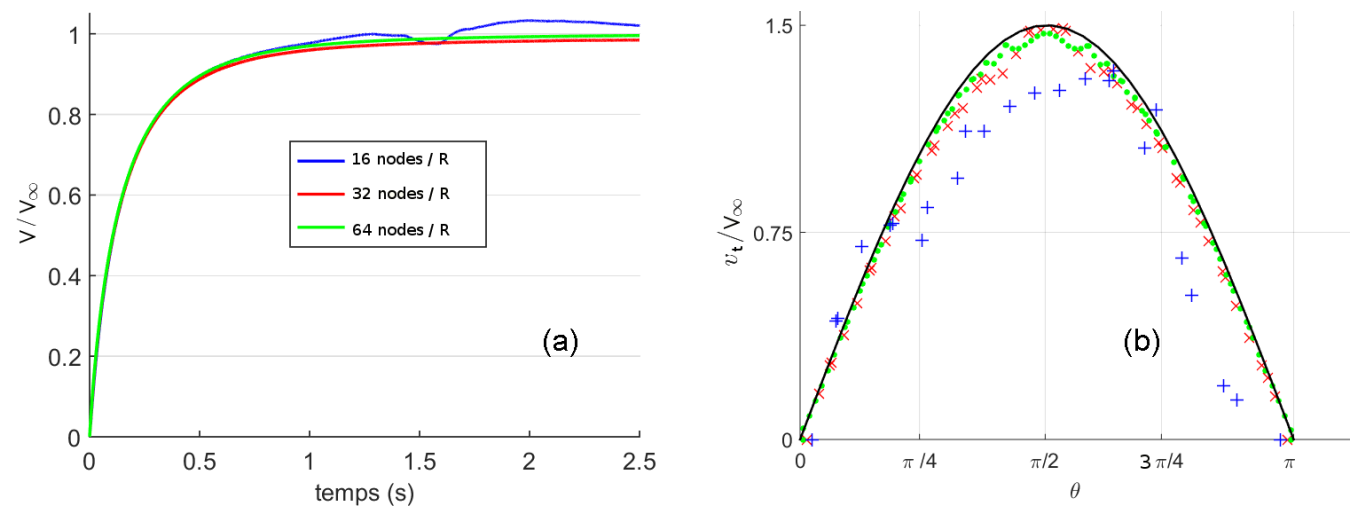

FIG. 2. Marangoni force test case. (a) Velocity of the drop against time, normalized by the prediction of the terminal velocity $V_{\infty}$ [by Eq. (15)] from the theory of Young et al. [35] in the Stokes regime. Simulation at $\mathrm{Re}_{\text {asc }}=0.08, \mathrm{We}=1.5 \times 10^{-4}$. (b) Tangential velocity at the interface at steady state for several mesh sizes (blue "+" 16 nodes per radius, red " $\times$ " 32 nodes per radius, green "." 64 nodes per radius) compared to the analytical profile of Hadamard and Rybczynski [36,37] (solid black line).

Except in the case of the coarser mesh, the numerical results are in very good agreement with the theoretical value, with a discrepancy of $5 \%$ for a resolution of 32 nodes per radius, and $3 \%$ for a resolution of 64 nodes per radius, demonstrating the mesh convergence of the calculation. In Fig. 2(b), the numerical profile of the tangential velocity $v_{t}$ along the interface is also compared to the analytical solution of Hadamard-Rybczynski [36,37], showing a good agreement for the two finer meshes.

\section{NUMERICAL RESULTS FOR CONTAMINATED DROPLETS}

Droplet dynamics with insoluble surfactants is investigated under the physical assumptions given in Sec. II. A droplet of $n$-heptane in water is considered (see physical properties in Table I), at constant ratios $\widehat{\rho}=0.68$ and $\widehat{\mu}=0.4$, and initially covered with a uniform concentration of surfactants $\bar{\Gamma}(t=0)$. Three configurations are studied: in case 1 , the droplet is initially deformed and the oscillation dynamics around the spherical shape is investigated in the absence of rising motion; in case 2, the drop is set in motion by gravity without initial deformation, and the effects of the distribution of surfactant along the interface upon the rising motion are studied; in case 3, the droplet is both rising and undergoing shape oscillations due to an initial deformation.

In cases where gravity induces a motion, the Weber number based on the droplet rising velocity remains negligible, hence inertia due to the rising motion is not able to deform the droplet. In cases 1 and 3 where shape oscillations are present, droplet deformation is initiated in the mode $n=2$ of the spherical harmonics, with a prolate shape; the amplitude of deformation is set to $a_{2}(t=0)=0.1$ [see Eq. (16) below], a value small enough to remain in the linear regime of oscillation (cf. Lalanne et al. [23]), which ensures that no other modes of deformation appear in time. At each instant, the droplet shape can be decomposed into the basis of the spherical harmonics in a frame attached to the droplet; because of the previous assumptions, this decomposition involves only mode 0 of amplitude

TABLE I. Physical parameters for the simulations of a drop of heptane in water.

\begin{tabular}{lccccc}
\hline \hline$R(\mathrm{~mm})$ & $\rho_{i}\left(\mathrm{~kg} / \mathrm{m}^{3}\right)$ & $\rho_{o}\left(\mathrm{~kg} / \mathrm{m}^{3}\right)$ & $\mu_{i}(\mathrm{mPa} \mathrm{s})$ & $\mu_{o}(\mathrm{mPa} \mathrm{s})$ & $\sigma_{c}(\mathrm{~N} / \mathrm{m})$ \\
\hline 0.579 & 684 & 1000 & 0.4 & 1.0 & 0.049 \\
\hline \hline
\end{tabular}


$R$, and mode 2 of amplitude $a_{2}(t)$ :

$$
r(\theta, t)=R\left\{1+a_{2}(t) P_{2}(\cos (\theta))\right\},
$$

where $(r, \theta)$ are the polar coordinates in a frame where the amplitude of mode 1 , describing the translation, cancels, and where $P_{2}(\cos (\theta))=\frac{1}{2}\left[3 \cos ^{2}(\theta)-1\right]$ is the Legendre polynomial of order 2. Note that in case 2 , all along the rising motion, $a_{2}(t) \ll 1$.

In this section, simulations are carried out with a mesh resolution of 45 nodes per undeformed drop radius, which is higher than the resolution of the test cases of Sec. IV due to necessity to solve accurately both the shape-oscillation dynamics (calculation of viscous damping is demanding) and the steep surface gradients of $\Gamma$ due to the drop rising motion.

\section{A. Case 1: Shape oscillations of a drop without gravity}

The drop oscillates around a mean spherical shape due to the initial deformation. Additionally to the density and viscosity ratios, this problem depends on the Reynolds number of oscillation $\operatorname{Re}_{\mathrm{osc}}=\sqrt{\rho_{o} \sigma_{e} R} / \mu_{o}$, and the elasticity number $E=\bar{R} T \bar{\Gamma}_{e} / \sigma_{e}$, where the subscript $e$ refers to the equilibrium state $\left(\sigma_{e}=\sigma_{c}-\bar{R} T \bar{\Gamma}_{e}\right)$, i.e., once the oscillations have been fully damped. Under the assumption of insoluble surfactants, $\bar{\Gamma}_{e}$ is determined from conservation of moles between the initial and final times: $S(0) \bar{\Gamma}(0)=4 \pi R^{2} \bar{\Gamma}_{e}$, where $S(0)$ is the surface of a prolate spheroid with semiaxes equal to $(0.95 R, 1.1 R)$ and $\bar{\Gamma}(0)$ the initial surface coverage. In these conditions, $\bar{\Gamma}_{e}$ and $\bar{\Gamma}(0)$ differ by only $0.1 \%$. In this study, $\operatorname{Re}_{\text {osc }}=168$ and $E$ is varied from 0.0025 to 1 , by changing $\bar{\Gamma}_{e}$ and $\sigma_{c}$ simultaneously in order to keep $\mathrm{Re}_{\mathrm{osc}}$ constant.

As the drop oscillates, $\Gamma$ varies due to both the advection by the tangential velocity at the interface and to the deformation of the interface, inducing gradients of surface concentration. The resulting Marangoni effect tends to smooth the profile of $\Gamma$, which tends towards an equilibrium state with a uniform surface coverage.

Shape oscillations are characterized by the time evolution of $a_{2}(t)=a_{2}(0) \cos \left(\omega_{2} t\right) \exp \left(-\beta_{2} t\right)$, with $\omega_{2}$ the angular frequency and $\beta_{2}$ the damping rate. These two timescales are derived from the simulations by decomposing the droplet shape into spherical harmonics in order to recover $a_{2}(t)$ [Eq. (16)]. Frequency and damping rate can then be compared to the predictions of the linear theory [24] under the same assumptions as those of the simulations. This theoretical solution shows that all variables can be described by mode 2 , oscillating at its characteristic frequency $\omega_{2}$. Note that, unlike the theory, the simulation contains a transient state ( 1 or 2 period of oscillations), which is not described by mode 2 and which is disregarded in the evaluation of $\omega_{2}$ and $\beta_{2}$. Figures 3(a) and 3 (b) show that the simulations predict accurate values of both the frequency and the damping rate, with a maximum error of $1 \%$ on $\omega_{2}$ and of $10 \%$ on $\beta_{2}$, using a numerical resolution of 45 nodes per drop radius. As in the case without surfactant (cf. Ref. [23]), the discrepancy is slightly higher on $\beta_{2}$ because it is a second-order magnitude quantity, which requires an accurate resolution of the fine boundary layers of oscillations that ensure the continuity of the tangential velocity across the interface. The good agreement of the simulation with the linear theory constitutes a substantial validation of the whole numerical method in the presence of insoluble surfactants.

It is observed in Fig. 3 that $\omega_{2}$ doesn't vary more than $5 \%$ compared to the case of clean drop ( $E=0)$, whereas the dependence of $\beta_{2}$ on $E$ is more significant and nonmonotonic: $\beta_{2}$ is almost unchanged when $E \leqslant 10^{-2}$, then abruptly increases and passes through a maximum close to 2.5 times the corresponding clean value when $E$ is around 0.1, then decreases and tends towards a limit (about 1.5 times the damping rate of a clean droplet) for larger values of $E$. Next we examine the variation of $\beta_{2}$ as a function of $E$ in more detail.

The damping rate is the characteristic time associated to the viscous dissipation of the mechanical energy of oscillation. The dissipation per unit of volume, $\psi$, is related to the rate of deformation tensor $\overrightarrow{\vec{D}}$ by the following equation:

$$
\psi=2 \mu \overrightarrow{\vec{D}}: \overrightarrow{\vec{D}}
$$



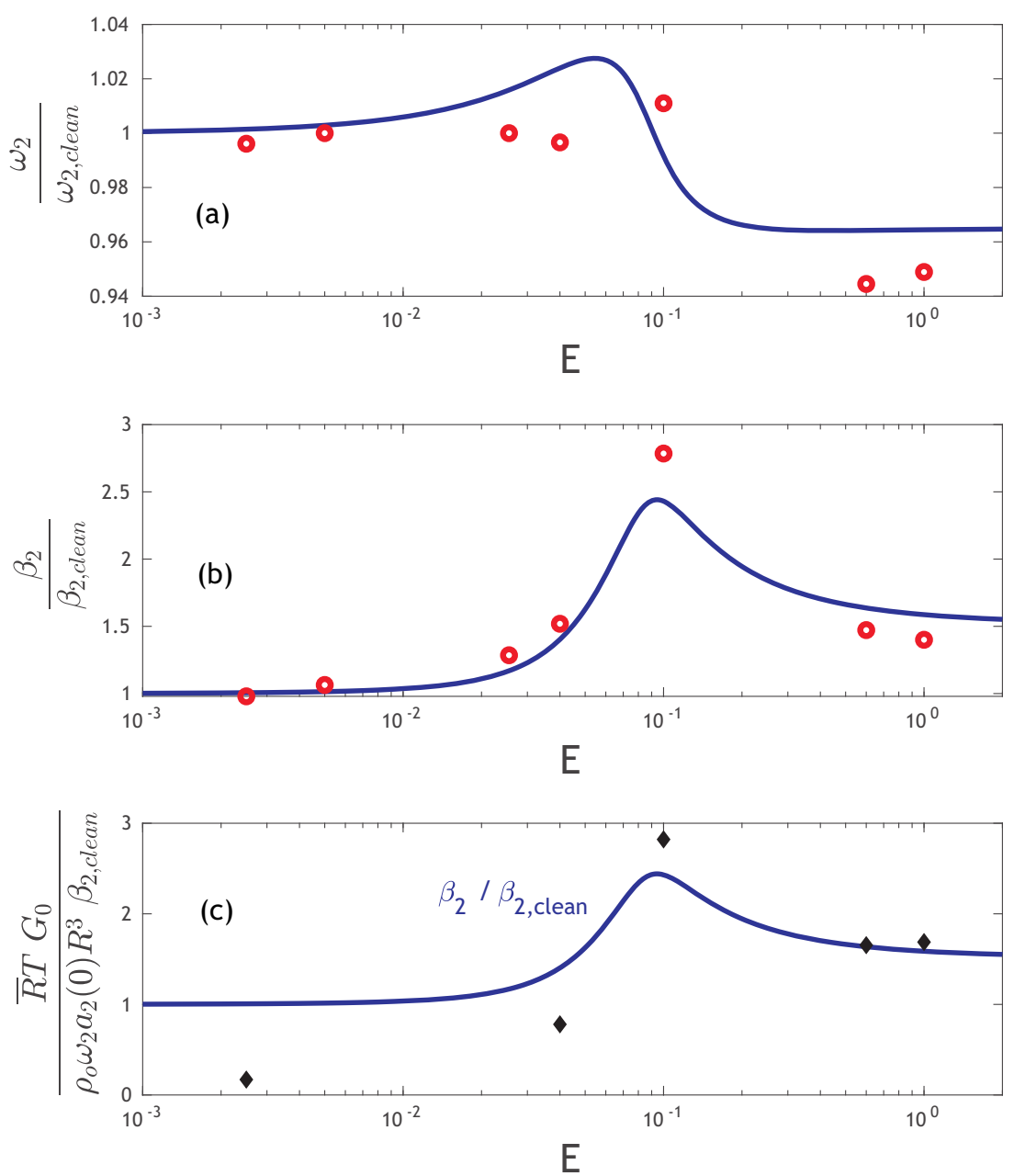

FIG. 3. Case 1: Comparison of numerical results (open circles), resolution of 45 nodes per radius, with the theoretical prediction of Lu and Apfel [24] (continuous line) of (a) frequency and (b) damping rate of oscillations of mode $n=2$, normalized by the corresponding values for a clean droplet $\omega_{2 \text {,clean }}$ and $\beta_{2 \text {,clean }}$. (c) Estimation of $\beta_{2} / \beta_{2 \text {,clean }}$ from Eq. (19) (closed diamonds) and comparison with the theoretical prediction of Lu and Apfel (continuous line).

Figure 4 shows the distribution of $\psi$ in the case of drops at different $E$ (and same $\operatorname{Re}_{\mathrm{osc}}$ ) and at an instant where the droplet has a spherical shape. For a clean drop, dissipation arises from two contributions: the major part comes from the boundary layers located on both sides of the interface, which represents about $80 \%$ of the damping; the remaining contribution is provided from the potential flow generated by the oscillating motion for which dissipation occurs mainly near the poles. In the presence of surfactants, due to the Marangoni effect, the contribution of the boundary layers in the total dissipation increases. Interestingly, it is observed that, as $E$ increases, the boundary layer moves progressively to the outer phase. This change in the location of dissipation can be quantified by integrating the dissipation over each fluid domain $\Omega, \Psi=\int_{\Omega} \psi d V$. Figure 5 displays the total dissipation $\Psi$ in each fluid phase as a function of $E$. On this graph, $\Psi$ is divided by twice the mechanical energy $E_{m}$ averaged over an oscillation cycle, so as to be directly comparable to the damping rate of the oscillations $\left(\beta_{2}=\frac{1}{2 E_{m}} \Psi\right)$. It can be verified in Fig. 5 that the normalized total dissipation follows the evolution of $\beta_{2}$ as a function of $E$, with a maximal value reached at $E=0.1$. 



FIG. 4. Case 1: Velocity and dissipation fields per unit volume at $t=2 T_{2}+T_{2} / 4$ (spherical shape). From left to right: $E=[0 ; 0.04 ; 0.1 ; 1]$. Note that units are arbitrary, and the values cannot be directly compared from one case to another since the amplitude of oscillation is not the same.

Contributions from each phase are also plotted on this graph; since the outer fluid is more viscous, the dissipation rate is always higher in the outer phase. It is noted again that, when $E$ increases, the dissipation increases in the outer phase but decreases in the inner phase. When $E>0.1$, almost no dissipation occurs inside the droplet, where the flow is transformed into a nearly irrotational flow.



FIG. 5. Case 1: Evolution of the dissipation $\Psi$ (calculated from the second oscillation period) as a function of $E$, normalized by the average mechanical energy during this period and the damping rate of the clean drop. 

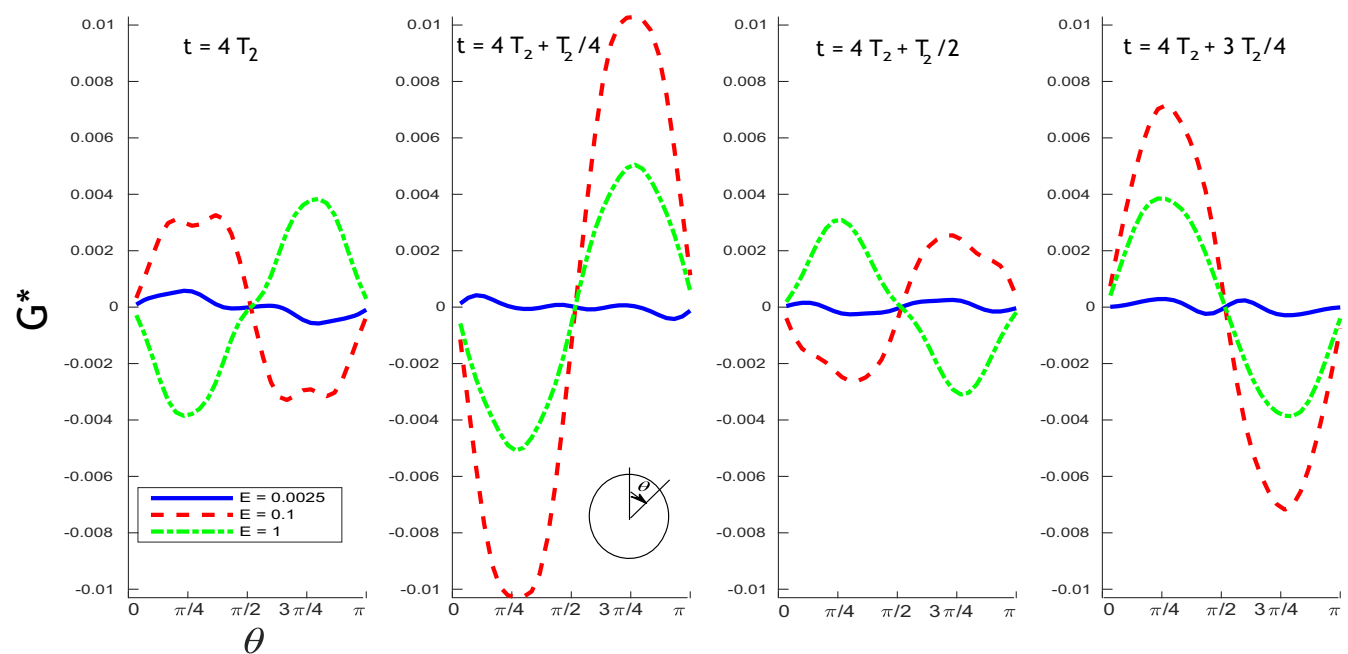

FIG. 6. Case 1: Profiles of normalized surface concentration gradient $G^{*}(\theta, t)$ at different instants in the oscillation cycle for $\mathrm{Re}_{\text {osc }}=168$ and various values of $E$. Blue [-]: $E=0.0025$, red [- - ]: $E=0.1$, green [--]: $E=1$. From left to right, $t=4 T_{2}$ (prolate), $t=4 T_{2}+T_{2} / 4$ (spherical), $t=4 T_{2}+T_{2} / 2$ (oblate), $t=$ $4 T_{2}+3 T_{2} / 4$ (spherical).

Denoting $\delta$ the characteristic boundary layer thickness associated to the oscillating motion in the outer (more viscous) phase, the dominant contribution in the dissipation arises from this boundary layer, leading to

$$
\Psi \sim \mu_{o}\left(\frac{U}{\delta}\right)^{2} V_{B L},
$$

where $U$ scales the oscillating velocity and $V_{B L}$ is the volume of the boundary layer, scaling as $V_{B L} \sim \pi R^{2} \delta$.

In the presence of surfactants, the magnitude of the tangential stress $\mu_{o} \frac{U}{\delta}$ at the interface is related to the Marangoni effect, which intensity during the oscillation process is scaled by the surface concentration gradient, $G(\theta, t)=\frac{\partial \Gamma(\theta, t)}{\partial \theta}$. Figure 6 displays the profiles along the interface of this nondimensional gradient, $G^{*}(\theta, t)=\frac{\bar{R} T G(\theta, t)}{\sigma_{e}}$, at different values of the Gibbs elasticity and at different stages of the oscillation cycle corresponding to following drop shapes: prolate (elongated), oblate (flattened), and spherical. When $E=0.0025$, the Marangoni effect is negligible and surfactant concentration can be considered as a passive scalar in this case. At $E=0.1$ and 1, gradients are of comparable intensity during the oblate and prolate phases, but when the droplet passes through the spherical shape, i.e., when all mechanical energy of oscillation is kinetic energy, the Marangoni effect is stronger at $E=0.1$ than at $E=1$. Hence it can be concluded that increasing the loading of surfactants at the interface doesn't necessarily increase the Marangoni interfacial force.

In each case, the maximum value $G_{M}^{*}(t)=\max _{\theta}\left|G^{*}\right|$ of the dimensionless gradient $G^{*}$ along the interface is recorded at each instant, these maxima being located as a general trend near $\theta=\frac{\pi}{4}$ and $\frac{3 \pi}{4}$, where the dissipation is maximum in the boundary layer. Figure 7 illustrates the time evolution of $G_{M}^{*}(t)$ in the case $E=0.04$. It can be observed that the envelope of $G_{M}^{*}$ oscillations accurately follows an exponential decay equal to $G_{0}^{*} e^{-\beta_{2} t}$. During the first oscillation, $G_{M}^{*}$ reaches either higher or lower values than in the pseudoperiodic regime because of the transient process in the simulation. To overcome this issue, we define the extrapolation of the envelope at $t=0$, denoted $G_{0}^{*}$, as the reference value of the gradient of $\Gamma$ for each studied case (the associated dimensional value is denoted $G_{0}=\frac{G_{0}^{*} \sigma_{e}}{\bar{R} T}$. 




FIG. 7. Case 1: Variations of the normalized maximum gradient of surface concentration $G_{M}^{*}$ at $\operatorname{Re}_{\mathrm{osc}}=$ 168 and $E=0.04$. Red line: exponential envelope $G_{M}^{*}(t)=G_{0}^{*} e^{-\beta_{2} t}$.

Using this reference value of the Marangoni effect intensity, the interface tangential stress can be scaled as $\mu_{o} \frac{U}{\delta} \sim \frac{\bar{R} T G_{0}}{R}$. Combined with Eq. (18), the total dissipation reads $\Psi \sim \pi \bar{R} T G_{0} U R$. From the definition of $\beta_{2}\left[=\psi /\left(2 E_{m}\right)\right]$ with $E_{m} \sim \frac{1}{2} \rho_{0} U^{2}\left(\frac{4}{3} \pi R^{3}\right)$, this scaling leads to the following relation between the damping rate and the intensity of the Marangoni effect:

$$
\beta_{2} \sim \frac{\bar{R} T G_{0}}{\rho_{o} U R^{2}} .
$$

Note that the density of the outer phase $\rho_{o}$ has been used to characterize the mass of the system, but this choice does not have a significant influence since densities of both phases are close (liquid-liquid system). This scaling is in agreement with the fact that $\beta_{2}$ does not change in time, since both the maximal value of the surface gradient of concentration, $G_{0}$, and the characteristic oscillating velocity, $U$, decay in time at the same rate $\beta_{2}$. The scaling of Eq. (19) has been tested in Fig. 3(c), taking $U \sim \omega_{2} a_{2}(0) R$ as the characteristic oscillating velocity, and compared to the values of $\beta_{2}$ issued from the simulations. The agreement is good, especially at moderate and large values of $E$, when the assumption of an external tangential viscous stress given by the Marangoni effect intensity is the most accurate. The interest of the relation (19) is to show that the damping rate of the oscillations is directly proportional to the intensity of the gradient of $\Gamma$ (Marangoni force) which is generated during the oscillation process. This allows us to understand the fact that the damping follows a nonmonotonic function of the Gibbs elasticity, as observed in some experimental works $[26,27]$ with shape-oscillating bubbles.

\section{B. Case 2: Rising motion without shape oscillations}

A spherical drop of heptane is rising in water under the effect of gravity, without oscillations of its shape, in the presence of surfactants. Additionally to the density and viscosity ratios, this problem depends on the Archimedes number $\mathrm{Ar}=\rho_{o} \sqrt{g d^{3}} / \mu_{o}$ ( $g$ being the gravity acceleration), the Weber number We $=\rho_{o} V_{\infty}^{2} R / \sigma_{e}$ based on the terminal velocity $V_{\infty}$, and the Marangoni number $\mathrm{Ma}=\bar{R} T \bar{\Gamma}(0) /\left(\mu V_{\infty}\right)$ which compares the Marangoni and viscous stresses at the interface. This study is carried out at $\mathrm{Ar}=123$ and in the limit of small Weber numbers (maximum value of We is 0.23 ), ensuring that the droplet remains approximately spherical all along its rising motion in all 

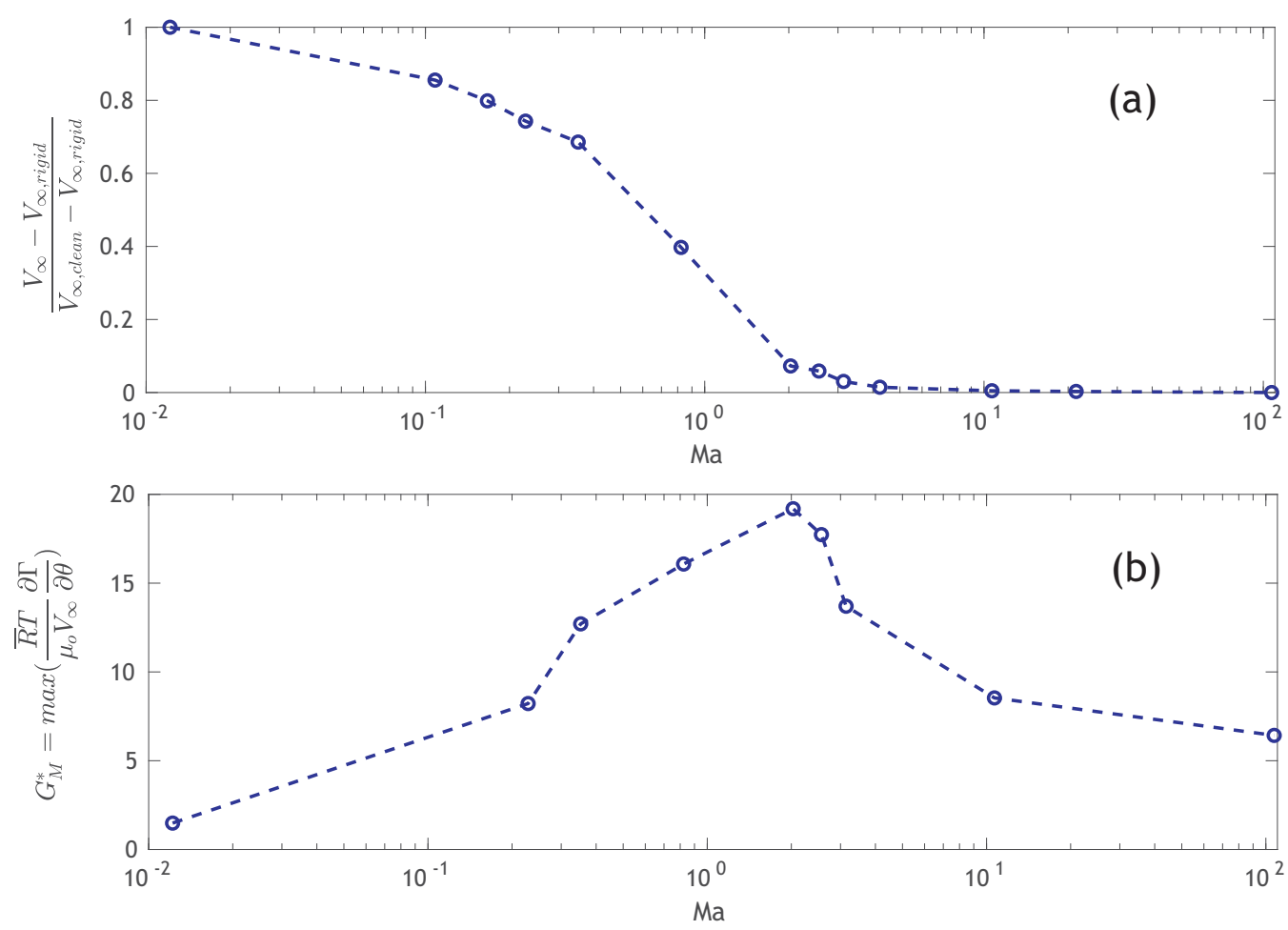

FIG. 8. Case 2: Evolutions as a function of the Marangoni number Ma at steady state: (a) dimensionless terminal velocity, (b) maximum value of the dimensionless gradient of the surface concentration $G_{M}^{*}$.

cases. Ma is varied in a wide range $(0.01 \leqslant \mathrm{Ma} \leqslant 100)$, and the Reynolds number of ascension $\operatorname{Re}_{\text {asc }}=2 \rho_{o} V_{\infty} R / \mu_{o}$ lies between 70 and 115 .

In order to analyze the rising velocity of the contaminated droplet as a function of Ma, two reference velocities are defined: $V_{\infty \text {,clean }}$, which is the theoretical velocity of a clean droplet (with an assumption of continuity of tangential stresses at the interface), and $V_{\infty}$,rigid, which is the velocity of the solid sphere of same size (with a no-slip condition at the interface). These two velocities are deduced from the force balance at equilibrium, $V_{\infty}=\sqrt{\frac{8}{3} \frac{\left(\rho_{o}-\rho_{i}\right)}{\rho_{o}} \frac{(R g)}{C_{d}}}$, with the drag coefficient $C_{d}$ given by the correlation of Rivkind and Ryskin [38] $C_{d \text {,clean }}=\frac{C_{d}^{\mathrm{b}}+\mu_{i} / \mu_{o} C_{d}^{\mathrm{s}}}{1+\mu_{i} / \mu_{o}}$ for the clean droplet, where $C_{d}^{b}=\frac{48}{\mathrm{Re}_{\text {asc }}}\left(1-\frac{2.21}{\mathrm{Re}_{\text {asc }}^{0.5}}\right)$ is the drag coefficient of a spherical clean bubble calculated by Moore [39], and $C_{d}^{\mathrm{s}}=\frac{24}{\operatorname{Re}_{\mathrm{ASC}}}\left(1+0.15 \operatorname{Re}_{\mathrm{ASC}}^{0.687}\right)$ is that of a rigid sphere given by Schiller and Naumann [40] $\left(V_{\infty}=V_{\infty, \text { clean }}\right.$ for $C_{d}=C_{d \text {,clean }}$ and $V_{\infty}=V_{\infty \text {,rigid }}$ for $\left.C_{d}=C_{d}^{\mathrm{s}}\right)$. In the simulations, when $\mathrm{Ma} \ll 1$, the velocity at steady state is very close to the theoretical value of a clean droplet. As Ma increases, the macroscopic consequence of the Marangoni interfacial stress is a decrease of the terminal velocity of the droplet compared to the clean case, which is shown in Fig. 8(a). When $\mathrm{Ma} \geqslant 2$, the terminal velocity nearly reaches a plateau which corresponds to the velocity of a solid particle, the minimum possible value. Note that with a numerical resolution of 45 nodes per drop radius, the final velocity matches the theoretical value within $1 \%$.

In the simulations, the initial distribution of surfactants at the interface is uniform, the drop being at rest. When the drop begins to rise, a concentration gradient develops due to the tangential flow, which is not surface divergence free. This flow sweeps the surfactants towards the rear of the droplet, building up a gradient of $\Gamma$ and hence a Marangoni force that tends to reduce it. Finally, an equilibrium between the downward advective flux of surfactants and the upward Marangoni flux is 

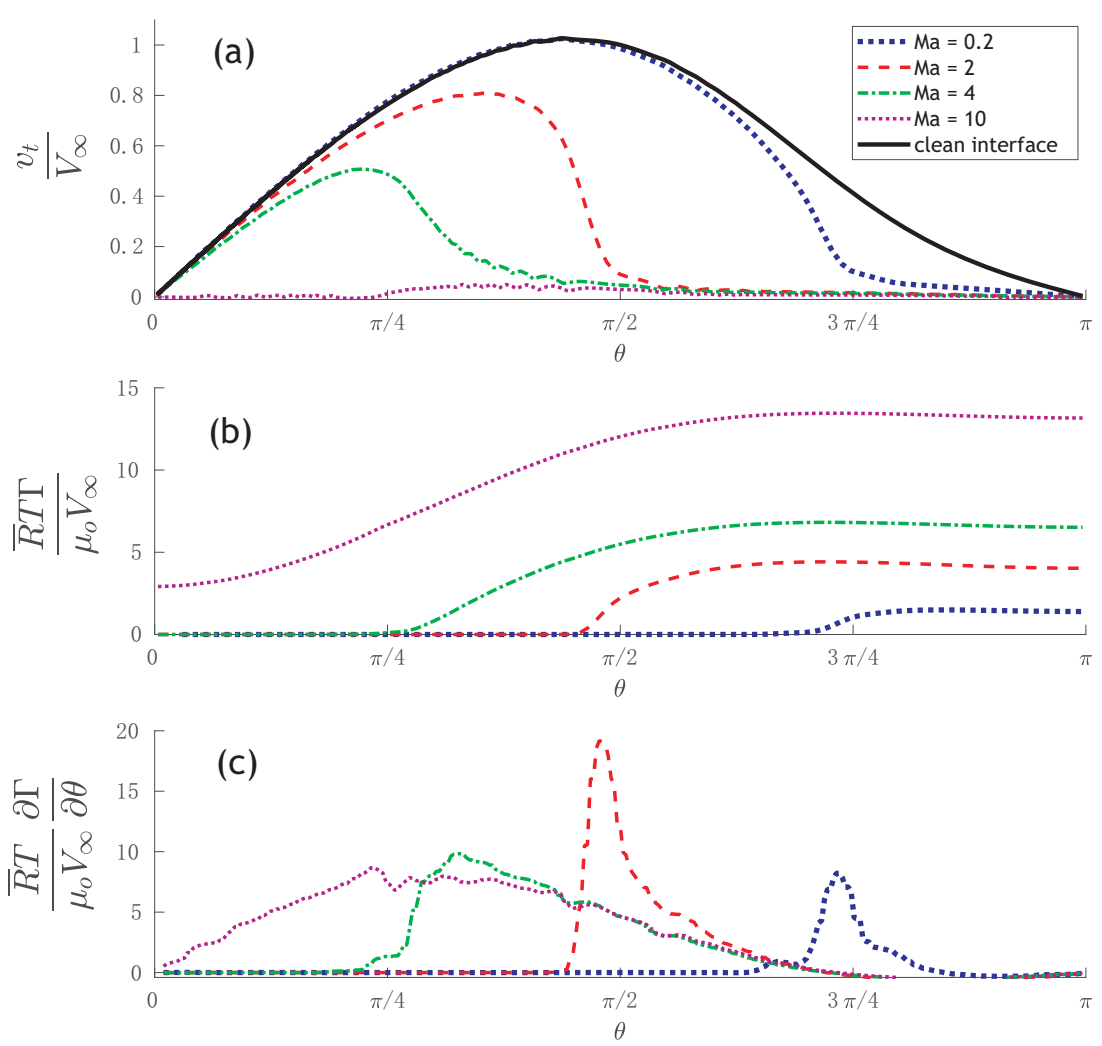

FIG. 9. Case 2: Evolutions along the interface at steady state at various Marangoni numbers: [...] Ma $=$ $0.2,[--] \mathrm{Ma}=2,[\cdot-\cdot] \mathrm{Ma}=4,[\cdots] \mathrm{Ma}=10$, and in (a) [-] clean interface. (a) tangential velocity (in the frame of the droplet), (b) nondimensional surface concentration, and (c) nondimensional gradient of concentration.

reached, leading to a steady state profile of $\Gamma$. It has been verified through a mesh convergence study on the profile of $\Gamma$ along the interface that the mesh resolution of 45 nodes per radius employed here is sufficient to compute accurately the strong gradient in this profile. Figures 9(a) and 9(b), respectively, display the tangential velocity along the interface in the droplet frame and the distribution of surfactant concentration, at steady state. For low values of Ma, all surfactant is distributed at the rear of the droplet, which corresponds to the zone where the tangential velocity vanishes due to Marangoni stress. As Ma is increasing [i.e., as increasing $\bar{\Gamma}(t=0)$, keeping constant all other parameters], the surfactant-free portion of the interface reduces while the portion with zero tangential velocity increases. For $\mathrm{Ma} \geqslant 10$, all the interface is covered by surfactants, and the tangential velocity vanishes over the whole interface. Regarding the rising motion, the drop behaves as a rigid particle. These profiles of $\Gamma$ and $v_{t}$ at steady state are consistent with the steady solution of Eq. (4), which can be simplified as $\vec{\nabla}_{s} \cdot(\Gamma \vec{v})=0$, leading to zones with either zero $v_{t}$ or $\Gamma$.

Generally, the existence of these two zones at the interface is emphasized by introducing the angle $\theta_{\text {cap }}$ in reference to the stagnant cap model firstly introduced by Savic [41]; note that, with this definition, it is considered that $\theta_{\text {cap }}=0$ in the case of a sufficiently high value Ma so that there is no part of the interface free of surfactant. Starting from a perfectly clean interface, as Ma is increasing, the progressive decrease of the terminal velocity can be quantified by introducing the reduced drag coefficient $C_{d}^{*}=\left(C_{d}-C_{d}^{\text {Clean }}\right) /\left(C_{d}^{\text {Rigid }}-C_{d}^{\text {Clean }}\right)$, which is displayed in Fig. 10 as a function of $\theta_{\text {cap }}$. On this graph, the analytical solution of Sadhal and Johnson [42] in the Stokes regime and the numerical predictions of Cuenot et al. [10] for a bubble at $\mathrm{Re}_{\text {asc }}=100$ have been also reported. The 




FIG. 10. Case 2: $C_{d}^{*}$ as a function of the contamination angle $\theta_{\text {cap }}$ for droplets at Reynolds numbers $O(100)$. Comparison with numerical results from Cuenot et al. [10] for bubbles at $\mathrm{Re}_{\mathrm{asc}}=100$, and with the analytical solution of Sadhal and Johnson [42] in the Stokes regime.

data from both simulations at similar $\mathrm{Re}_{\mathrm{asc}}$ are very close and differ only slightly from the theory in Stokes conditions, showing that the decrease of velocity due to the Marangoni effect is weakly sensitive to both the rising regime and the nature of the fluid particle (droplet or bubble). In this figure, it is noted that the terminal velocity of a contaminated drop or bubble can be very close to that of a solid particle (i.e., $C_{d}^{*}$ close to 1 ), even though a significant part of the interface is free of surfactant (i.e., $\theta_{\text {cap }} \neq 0$ ), and thus there is only a portion of the interface which is fully immobilized (with zero tangential velocity), as is the case at $\mathrm{Ma}=2$.

At $\mathrm{Ma}=4$, Fig. 11 illustrates the velocity and dissipation fields compared to the clean case $(\mathrm{Ma}=0)$. This figure underlines the change of the recirculating vortex inside the droplet, which is strongly reduced compared to a clean drop, as also noticed in the experiments of Horton et al. [43]
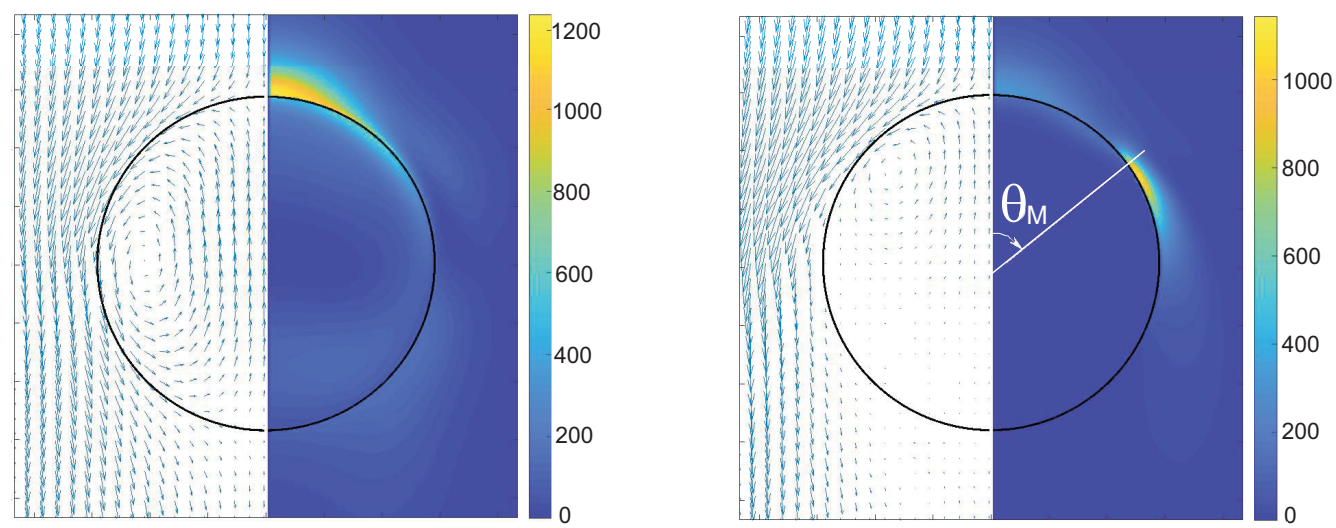

FIG. 11. Case 2: Velocity field (in the droplet frame) and dissipation field (arbitrary units) in the case of a rising droplet at $\mathrm{Ma}=0$ (left) and $\mathrm{Ma}=4$ (right), where the location of the angle $\theta_{M}$ of the maximum of surface gradient of concentration is shown (note that $\theta_{M}=\theta_{\text {cap }}$ for this Marangoni number value). 
and Hosokawa et al. [9], and in the simulations of Oguz and Sadhal [44] using the stagnant-cap model. Figure 11 also shows that, contrary to the clean case where viscous dissipation is maximal at the stagnant point at the front of the droplet, in the contaminated case, dissipation is maximal at the angle corresponding to the maximum value of the Marangoni stress.

In order to quantify the importance of the Marangoni effect, Fig. 9(c) shows the nondimensional gradient of $\Gamma$ defined as a local Marangoni number $\operatorname{Ma}^{*}(\theta)=\frac{\bar{R} T}{\mu V_{\infty}} \frac{\partial \Gamma}{\partial \theta}$, for different Ma and at steady state. On each profile, this gradient is observed to be maximum at $\theta_{\text {cap }}$ in the case where two distinct zones exist on the interface, whereas it is located at a converged position at high Ma values, for which the whole interface is covered with surfactants. Surprisingly, it is observed that the maximal intensity of this gradient does not evolve monotonically with Ma. Denoting $G_{M}^{*}=\max _{\theta}\left|\mathrm{Ma}^{*}\right|$ as the maximal nondimensional value of this gradient along the interface, the evolution of $G_{M}^{*}$ as a function of $\mathrm{Ma}$ is reported in Fig. 8(b). It is observed that the Marangoni effect is maximum for $\mathrm{Ma}=2$, and then decreases as Ma is further increased.

At the interface, the Marangoni stress counterbalances the shear stress induced by the motion of the droplet, so that the tangential velocity $v_{t}$ is much smaller and the advection of surfactant is considerably reduced. If we denote $\theta_{M}$ the angular location of Marangoni stress maximum value, we observe that the tangential velocity is close to zero at $\theta_{M}$ and the velocity inside the droplet also vanishes for $\theta \geqslant \theta_{M}$ [Fig. 9(a)], leading to a negligible internal stress at this position. So this maximum can be scaled by the viscous stress exerted by the sole external flow, i.e., $\max _{\theta}\left(\bar{R} T \frac{1}{R} \frac{\partial \Gamma}{\partial \theta}\right)=\mu_{o} \frac{\partial v_{t}}{\partial n} \sim \mu_{o} \frac{V_{\text {pot }}\left(\theta_{M}\right)}{\delta}$, where $\delta$ is the thickness of the boundary layer and $V_{\text {pot }}\left(\theta_{M}\right)$ is the potential flow velocity around a sphere evaluated at the drop surface at the angle $\theta_{M}$ (we remind readers that $\theta_{M}$ corresponds to $\theta_{\text {cap }}$ for Ma $\leqslant 4$ but that they have different values at higher Ma), leading to a maximal nondimensional gradient $G_{M}^{*}$ scaling as

$$
G_{M}^{*} \sim \frac{V_{\text {pot }}\left(\theta_{M}\right)}{V_{\infty}} \frac{R}{\delta} .
$$

As Ma increases, the location $\theta_{M}$ moves towards the front of the droplet, hence the evolution of $G_{M}^{*}(\mathrm{Ma})$ is similar to that of $V_{\text {pot }}\left(\theta_{M}\right)$, reaching a maximum at $\mathrm{Ma}=2$ where $\theta_{M}$ corresponds to the droplet equator, and decreasing beyond. For large values of Ma $[O(10)]$, the droplet internal motion totally vanishes and $\theta_{M}$ converges towards $\pi / 4$, explaining why $G_{M}^{*}$ tends towards a finite asymptotic value at large Ma.

\section{Case 3: Coupling between shape oscillations and rising motion}

We consider now a rising droplet, with shape oscillations of low amplitude. Additionally to the density and viscosity ratios, the problem is described by the elasticity number $E$, the Marangoni number Ma, and the Archimedes number Ar, which is kept constant at 123. E and Ma compare the elasticity associated to the surface concentration gradients respectively induced by the shapeoscillating motion and by the rising motion. Simulations have been performed in the range $0.0025 \leqslant$ $E \leqslant 1$ and $2 \leqslant$ Ma $\leqslant 430$ by changing only $\bar{\Gamma}(0)$, all other physical parameters remaining constant (see Table II). This range of parameters corresponds to both large rising and oscillating Reynolds numbers $\left(65 \leqslant \operatorname{Re}_{\text {asc }} \leqslant 70\right.$ and $\left.\operatorname{Re}_{\text {osc }}=168\right)$ and to a low Weber number (We $\left.\leqslant 0.1\right)$, ensuring that the droplet keeps a spherical shape in average.

Figure 12 compares the time evolution of the instantaneous ascension Reynolds number for an oscillating and a nonoscillating droplet at $\mathrm{Ma}=2$. For the oscillating drop, the rising velocity also exhibits small amplitude oscillations at a frequency $\omega_{2}$, around the velocity of the nonoscillating droplet. These oscillations are due to an added-mass effect induced by the periodic variations of the drop shape (oblate-to-prolate oscillation). Note that this slight influence of the shape oscillations on the rising velocity has been already observed by De Vries et al. [45] and Lalanne et al. [23].

As seen in previous sections, for the shape-oscillations as well as for the translating motion, dissipation of energy is driven by the magnitude of the gradient of surface concentration which 
TABLE II. Case 3: Adimensional parameters for the simulations of a rising and shape-oscillating drop of heptane in water with surfactants.

\begin{tabular}{lccc}
\hline \hline Case & $\mathrm{A}$ & $\mathrm{B}$ & $\mathrm{C}$ \\
\hline $\mathrm{Ma}$ & 2 & 65 & 430 \\
$E_{s}$ & 0.0025 & 0.1 & 1 \\
$\mathrm{Re}_{\text {osc }}$ & 168 & 168 & 168 \\
$\mathrm{Re}_{\text {asc }}$ & 69.5 & 65.9 & 65.8 \\
\hline \hline
\end{tabular}

develops at the interface. Figure 13 plots the time evolution of this quantity normalized as $G_{M}^{*}(t)=$ $\bar{R} T \max _{\theta}|G(\theta, t)| / \sigma_{e}$, in the case $(E=0.0025, \mathrm{Ma}=2)$; the curve is compared to the evolution of this gradient in the case of shape oscillations only (at $E=0.0025$ and same $\mathrm{Re}_{\mathrm{OSC}}$ ) and in the case of rising drop without oscillations ( $\mathrm{Ma}=2$ and same $\mathrm{Ar}$ ). The value of $G_{M}^{*}(t)$ is observed to increase until reaching the terminal state. After the first period, the evolution of $G_{M}^{*}(t)$ for the shapeoscillating and rising droplet follows that of the rising-only droplet, with an identical position of the maximum along the interface. At terminal velocity, the value of $G_{M}^{*}(t)$ induced by the drop translation is much larger than that induced by the shape oscillation at the beginning of the simulation.

The shape oscillation dynamics is displayed in Fig. 14 where the maximum of the absolute value of the oscillation amplitude, $\left|a_{2}\right|$, has been plotted as a function of time for three sets of parameters ( $E, \mathrm{Ma})$. (Note that the slope of each curve gives the damping coefficient $\beta_{2}$ in each case). On this graph, the evolution of $\left|a_{2}\right|$ has been compared to that predicted by the linear theory of Lu and Apfel [24], which disregards the rising motion. In all cases covering a wide range of $E$ and Ma, it is observed that the values of $\omega_{2}$ and $\beta_{2}$ are not affected by the rising motion.

Hence, although the maximal value of the surface concentration gradient is dominated by the rising motion, the drop shape oscillates as if the rising motion was absent, with a damping rate depending only upon the elasticity number $E$, provided that the average droplet shape remains spherical in time $(\mathrm{We} \ll 1)$.

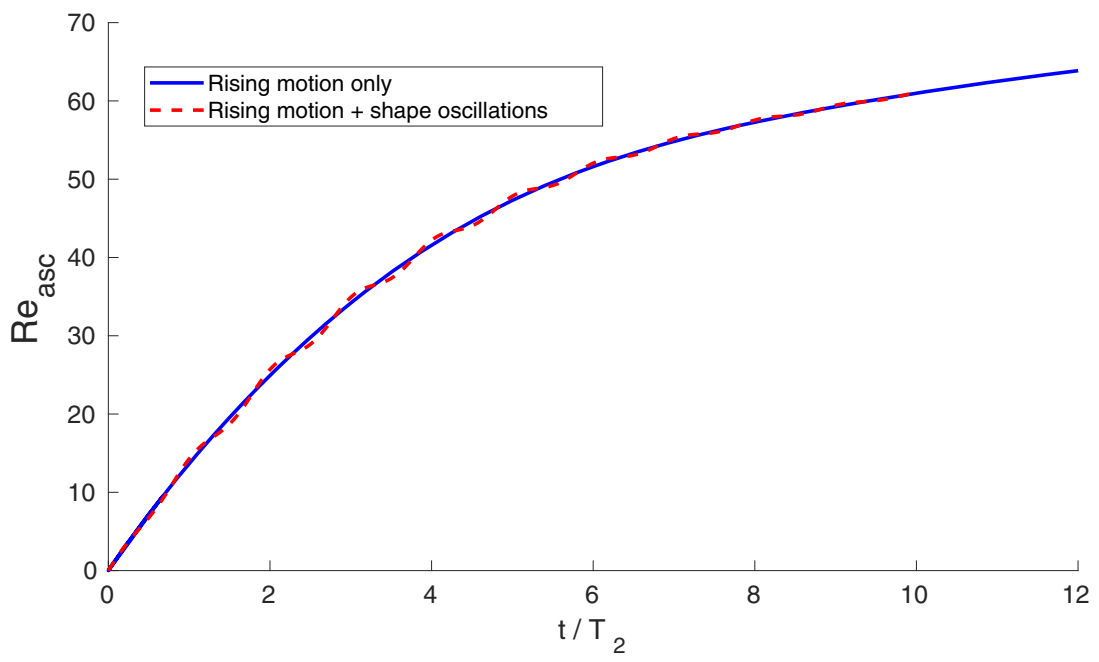

FIG. 12. Cases 2 and 3: Time evolution of the rising droplet Reynolds number at $\mathrm{Ma}=2$, without shape oscillations (continuous blue line) and with shape oscillations (dashed red line) (parameters of case A in Table II). 


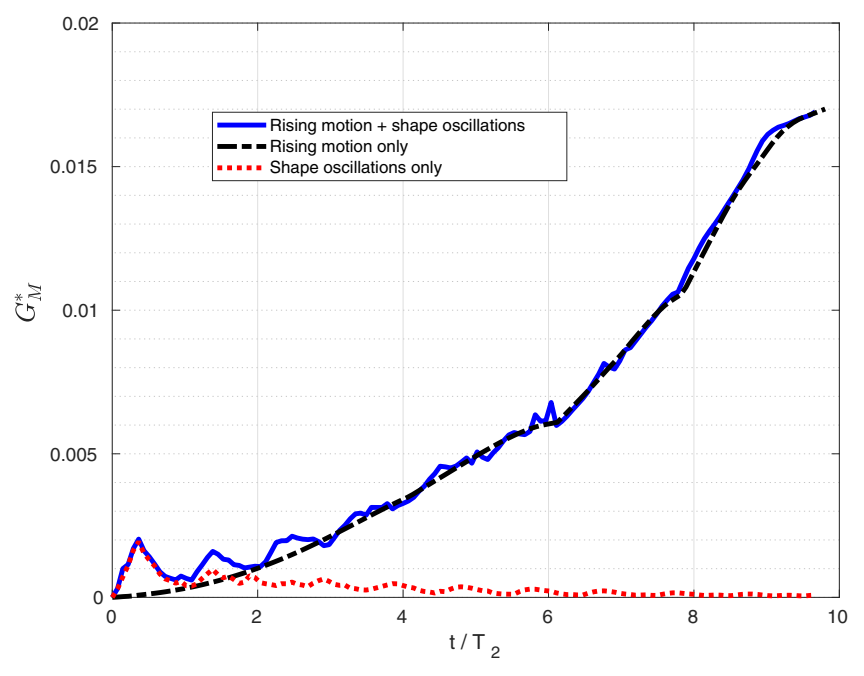

FIG. 13. Cases 1,2, and 3: Time evolution of the spatial maximum value $G_{M}^{*}$ of the normalized gradient of surface concentration. Rising and shape-oscillating droplet: $E=0.0025, \mathrm{Ma}=2\left(\operatorname{Re}_{\mathrm{osc}}=168\right.$ and $\left.\mathrm{Ar}=123\right)$. Rising droplet only: $\mathrm{Ma}=2$ and $\mathrm{Ar}=123$. Shape-oscillating droplet only: $E=0.0025$ and $\mathrm{Re}_{\mathrm{osc}}=168$.

A particularly striking example is the case $(\mathrm{Ma}=2, E=0.0025)$, for which the droplet has a rising velocity close to that of a rigid particle but oscillates as a clean drop. This surprising result (shown here in the linear regime of oscillation) is the numerical confirmation of an experimental observation realized by Abi Chebel et al. [28] (in conditions of moderate nonlinear shape oscillation). In that study, the authors explained the value of the drop rising velocity (close to that of a rigid particle) by a residual contamination, despite the care taken to clean the experiment. However, the shape oscillation dynamics was found to be in agreement with the theory in the case of a clean nonrising droplet. The present numerical study shows that the case $\left(\mathrm{Ma}=2, E_{s}=0.0025\right)$

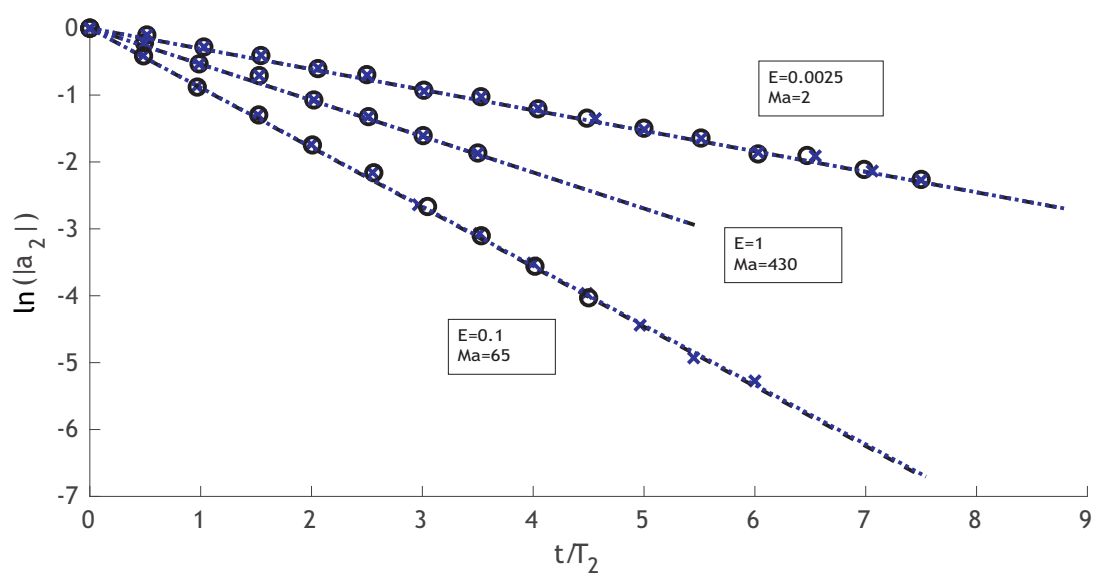

FIG. 14. Cases 1 and 3: Evolution of the maximum value of $\left|a_{2}\right|$ over time, for rising and shape-oscillating droplets (Case 3, blue crosses and dotted lines) and for a shape-oscillating droplet only (Case 1, black circles and dashed lines). Time is normalized by $T_{2}$, which is the theoretical period of oscillation of mode 2 predicted by the theory of Lu and Apfel [24]. The slope of the lines gives the value of the damping rate $\beta_{2}$, which are very close with or without rising motion. 
matches such conditions. The theory of Lu and Apfel can therefore be used to determine the range of elasticity number for which the effect of contamination on shape oscillations of a rising droplet can be neglected.

\section{CONCLUSION}

In this study, the presence of insoluble surfactants adsorbed at liquid-liquid interfaces in dilute concentration has been implemented in numerical simulations, and their effect on the droplet dynamics has been investigated.

Numerical results of oscillating drops without gravity show a good agreement with the theory of $\mathrm{Lu}$ and Apfel [24], which predicts the frequency and damping rate of linear oscillations. In the presence of surfactants, these two characteristic timescales depend on the elasticity number $E$, and compared to perfectly clean conditions, the frequency is nearly not affected by the presence of surfactant, whereas the damping rate can be increased significantly. However, the increase of damping does not evolve monotonically with the surfactant concentration (i.e., with $E$ ), as already revealed by experiments on bubble oscillations. We have shown here that this evolution is correlated to the nonmonotonic evolution of the maximum value of the surface concentration gradient induced by the oscillating motion.

The rising motion at low Weber number of a contaminated droplet has been investigated, introducing a Marangoni number to quantify the effect of surfactants on the rising motion. At low $\mathrm{Ma}$, and at steady state, part of the interface remains free of surfactants, which are advected towards the rear of the droplet where the tangential velocity vanishes because of the upward Marangoni flux. At large Ma, the whole interface is covered with surfactants. However, it has been observed that the droplet rising velocity can match that of a solid sphere although a significant part of the interface is surfactant free $\left(\theta_{\text {cap }}\right.$ close to $\left.3 \pi / 8\right)$. The intensity of the Marangoni effect does not evolve monotonically with the surfactant concentration (i.e., with Ma). An interpretation of this evolution is proposed here. As Ma increases, the Marangoni effect intensity increases as well, since the location of the maximum of the gradient along the interface moves towards the drop equator where the tangential velocity of the potential flow of reference is maximum. The intensity of the Marangoni stress then decreases at larger Ma, towards an asymptotic value that can probably be scaled using the solution of the flow around a solid particle.

The coupling between the rising motion and the shape oscillation dynamics has then been investigated in the limit of small Weber number. The gradient of surface concentration created by the oscillation is still characterized by $E$ and that produced by the rising motion is still characterized by Ma. It has been concluded, similarly to the case without surfactant, that gravity does not modify the frequency and the damping rate of the oscillation, even though the concentration profile is mainly driven by the rising motion. As a consequence, the theory of Lu and Apfel [24] is still able to predict the shape oscillation dynamics $\left(\omega_{2}\right.$ and $\left.\beta_{2}\right)$ of contaminated rising droplets. This is an important conclusion, useful for the interpretation of experimental results on oscillating droplets or bubbles moving in another liquid. It is valid provided (i) the oscillation amplitude is low enough so as to remain close to the linear regime of oscillation (discrepancy due to nonlinear effects is lower to $10 \%$ on the damping rate for amplitudes of $20 \% R$ [18]), (ii) the concentration of adsorbed surfactants is small compared to conditions of interface saturation, and (iii) the Weber number based on the rising velocity is small enough to maintain a spherical equilibrium drop shape. Note that at high Weber number, it is expected that gravity may have an influence on the oscillation dynamics by modifying the eigenmodes of oscillation due to an average drop deformation, similarly to what has been observed in the case of perfectly clean interfaces [23].

In particular, the present simulations explain why it has been possible to observe, in Abi Chebel et al. [28], a contaminated droplet rising as a rigid particle with a shape oscillation dynamics identical to that of a clean drop: Ma was sufficiently high and $E$ sufficiently low to match these conditions, leading to this unexpected fact. Note that this case has been simulated here by using the simplest possible model of a dilute insoluble surfactant, which confers only a Gibbs elasticity to 
the interface. In a real experiment, these conditions imply that the rates of desorption, adsorption of the surfactant between the interface, and the bulk fluids must be negligible compared to the oscillation frequency. Surfactant can be colloidal species or nanoparticles irreversibly adsorbed on the interface, as was probably the case in the experiment of Abi Chebel et al. [28].

\section{ACKNOWLEDGMENTS}

The authors thank both the COMUE of University of Toulouse and the Occitanie Region for providing the funding of this work.

[1] S. Takagi and Y. Matsumoto, Surfactant effects on bubble motion and bubbly flows, Annu. Rev. Fluid Mech. 43, 615 (2011).

[2] R. M. Edge and C. D. Grant, The terminal velocity and frequency of oscillation of drops in pure systems, Chem. Eng. Sci. 27, 1709 (1972).

[3] R. Bel Fdhila and P. C. Duineveld, The effect of surfactant on the rise of a spherical bubble at high Reynolds and Péclet numbers, Phys. Fluids 8, 310 (1996).

[4] A. Frumkin and V. Levich, On surfactants and interfacial motion, Zhur. Fiz. Khim. 21, 1183 (1947).

[5] Y. Tagawa, S. Takagi, and Y. Matsumoto, Surfactant effect on path instability of a rising bubble, J. Fluid Mech. 738, 124 (2014).

[6] F. H. Garner and A. Hale, The effect of surface active agents on liquid extraction processes, Chem. Eng. Sci. 2, 157 (1953).

[7] F. H. Garner and H. Skelland, Some factors affecting droplet behavior in liquid-liquid systems, Chem. Eng. Sci. 17, 1057 (1955).

[8] P. C. Duineveld, Bouncing and coalescence of bubble pairs rising at high Reynolds number in pure water or aqueous surfactant solutions, Appl. Sci. Res. 58, 409 (1998).

[9] S. Hosokawa, K. Masukura, K. Hayashi, and A. Tomiyama, Evaluation of adsorption of surfactant at a moving interface of a single spherical drop, Intl. J. Multiphase Flow 97, 157 (2017).

[10] B. Cuenot, J. Magnaudet, and B. Spennato, The effects of slightly soluble surfactants on the flow around a spherical bubble, J. Fluid. Mech. 339, 25 (1997).

[11] M. Drumright-Clarke and Y. Renardy, The effect of insoluble surfactant at dilute concentration on drop breakup under shear with inertia, Phys. Fluids 16, 14 (2004).

[12] A. J. James and J. Lowengrub, A surfactant-conserving volume-of-fluid method for interfacial flows with insoluble surfactant, J. Comp. Phys. 201, 685 (2004).

[13] J. J. Xu and H. K. Zhao, An Eulerian formulation for solving partial differential equations along a moving interface, J. Sci. Comput. 19, 573 (2003).

[14] C. C. De Langavant, A. Guittet, M. Theillard, F. Temprano-Coleto, and F. Gibou, Level-set simulations of soluble surfactant driven flows, J. Comput. Phys. 348, 271 (2017).

[15] M. Muradoglu and G. Tryggvason, A front-tracking method for computation of interfacial flows with soluble surfactants, J. Comput. Phys. 227, 2238 (2008).

[16] B. Lalanne, L. R. Villegas, S. Tanguy, and F. Risso, On the computation of viscous terms for incompressible two-phase flows with Level Set/Ghost Fluid Method, J. Comput. Phys. 301, 289 (2015).

[17] N. Abi Chebel, F. Risso, and O. Masbernat, Inertial modes of a periodically forced buoyant drop attached to a capillary, Phys. Fluids 23, 102104 (2011).

[18] B. Lalanne, N. Abi Chebel, J. Vejrazka, S. Tanguy, O. Masbernat, and F. Risso, Non-linear shape oscillations of rising drops and bubbles: Experiments and simulations, Phys. Fluids 27, 123305 (2015).

[19] J. W. S. Rayleigh, On the capillary phenomena of jets, Proc. R. Soc. 29, 71 (1879).

[20] H. Lamb, Hydrodynamics (Cambridge University Press, Cambridge, 1932).

[21] C. A. Miller and L. E. Scriven, The oscillations of a fluid drop immersed in another fluid, J. Fluid Mech. 32, 417 (1968). 
[22] A. Prosperetti, Normal mode analysis for the oscillations of a viscous liquid drop in an immiscible liquid, J. Mec. 19, 149 (1980).

[23] B. Lalanne, S. Tanguy, and F. Risso, Effect of rising motion on the damped shape oscillations of drops and bubbles, Phys. Fluids 25, 112107 (2013).

[24] H. L. Lu and R. Apfel, Shape oscillations of drops in the presence of surfactants, J. Fluid. Mech. 222, 351 (1991).

[25] Y. Tian, R. G. Holt, and R. E. Apfel, Investigation of liquid surface rheology of surfactant solutions by droplet shape oscillations: Experiments, J. Colloid Interface Sci. 187, 1 (1997).

[26] M. Corti, M. Pannuzzo, and A. Raudino, Out of equilibrium divergence of dissipation in an oscillating bubble coated by surfactants, Langmuir 30, 477 (2014).

[27] T. J. Asaki, D. B. Thiessen, and P. L. Marston, Effect of an Insoluble Surfactant on Capillary Oscillations of Bubbles in Water: Observation of a Maximum in the Damping, Phys. Rev. Lett. 75, 2686 (1995).

[28] N. Abi Chebel, J. Vejrazka, O. Masbernat, and F. Risso, Shape oscillations of an oil drop rising in water: effect of surface contamination, J. Fluid. Mech. 702, 533 (2012).

[29] V. G. Levich, Physicochemical Hydrodynamics (Prentice-Hall, New York, 1962).

[30] H. Stone, A simple derivation of the time-dependent convective-diffusion equation for surfactant transport along a deforming interface, Phys. Fluids A 2, 111 (1990).

[31] M. Kang, R. P. Fedkiw, and X.-D. Liu, A boundary condition capturing method for multiphase incompressible flow, J. Sci. Comput. 15, 323 (2000).

[32] M. Sussman, P. Smereka, and S. Osher, A level set approach for computing solutions to incompressible two-phase flow, J. Comput. Phys. 114, 146 (1994).

[33] Å. Ervik, T. E. Penne, S. M. Helles $\varnothing$, S. T. Munkejord, and B. Mueller, Influence of surfactants on the electrohydrodynamic stretching of water drops in oil, Int. J. Multiphase Flow 98, 96 (2018).

[34] K. Hayashi and A. Tomiyama, Effect of numerical treatment of viscous and surface tension forces on predicted motion of interface, J. Comp. Multiphase Flows 6, 111 (2014).

[35] N. Young, J. Goldstein, and M. Bock, The motion of bubbles in a vertical temperature gradient, J. Fluid Mech. 6, 350 (1959).

[36] J. Hadamard, Motion of liquid drops (viscous), Comp. Rend. Acad. Sci. Paris 152, 1735 (1911).

[37] W. Rybczynski, Uber die fortschreitende Bewegung einer flussigen Kugel in einem zahen Medium, Bull. Acad. Sci. Cracovie A 1, 40 (1911).

[38] V. Y. Ryvkind and G. Ryskin, Flow structure in motion of a spherical drop in a fluid medium at intermediate Reynolds number, Fluid. Dyn. 11, 5 (1976).

[39] D. W. Moore, The boundary layer on a spherical gas bubble, J. Fluid Mech. 16, 161 (1963).

[40] L. Schiller and A. Naumann, A drag coefficient correlation, Vdi Zeitung 77, 318 (1935).

[41] P. Savic, Circulation and distortion of liquid drops falling through a viscous medium, Technical Report MT-22. National Resource Council of Canada, Division of Mechanical Engineering (1953).

[42] S. Sadhal and R. Johnson, Stokes flow past bubbles and drops partially coated with thin films, J. Fluid. Mech. 126, 237 (1987).

[43] T. J. Horton, T. R. Fritsch, and R. C. Kintner, Experimental determination of circulation velocities inside drops, Can. J. Chem. Eng. 43, 143 (1965).

[44] H. N. Oguz and S. S. Sadhal, Effects of soluble and insoluble surfactants on the motion of drops, J. Fluid Mech. 194, 563 (1988).

[45] J. de Vries, S. Luther, and D. Lohse, Induced bubble shape oscillations and their impact on the rise velocity, Eur. Phys. J. B 29, 503 (2002). 